\title{
REVIEW ON MULTI-AGENT SYSTEM COLLABORATION IN LEARNING MANAGEMENT SYSTEM DOMAIN BY DEPLOYING WIRELESS SENSOR NETWORKS FOR STUDENT LOCATION DETECTION
}

\author{
${ }^{1}$ Mwinyi, A.K., ${ }^{1}$ S.A.R. AL-Haddad, ${ }^{1}$ S.J. Bin Hashim and ${ }^{2}$ R. Bin Hj Abdullah \\ ${ }^{1}$ Department of Computer and Communication Systems Engineering, Faculty of Engineering, \\ ${ }^{2}$ Department of Information System, Faculty of Computer Science and Information Technology, \\ Universiti Putra Malaysia, 43400 UPM Serdang, Selangor Darul Ehsan, Malaysia
}

Received 2013-12-19; Revised 2014-01-13; Accepted 2014-01-30

\begin{abstract}
Student location detection in Learning Management System (LMS) by utilizing Multi-Agent System (MAS) which contains sensor nodes is a new area of research. This study reviews several studies to ascertain the potential of integrating these two technologies to automate students' class attendance in Higher Learning Institutions (HLIs). Currently, the HLIs are using paper-based process to record students' attendance in the class, that is time consuming and is not possible to monitor students all the time, that they suppose to be in learning environment. Introducing the sensor networks and MAS in LMS system is to enable the instructors or lecturers to be aware of the presence of their students once they reach the system's domain. The collaboration using MAS facilitates the retrieval and recording of students' details from the sensors and then sends them to LMS servers through Cluster Head Sensor. The information that is collected and recorded by the agents include the signal strength of the students' device and their profiles which can facilitate to know the exactly locations of the students, by comparing such information with the information already stored in LMS database. Therefore, a system architecture that comprises MAS with sensor networks in LMS is presented in this study for monitoring students' attendance in the classes and labs. This type of system architecture with improved LMS features is more focused and intended for HLIs that follow the blended learning system. This proposed system has potential of boosting learning process in HLIs by providing new feature in LMS that monitor students' activities in blended systems, that support classroom and online teachings.
\end{abstract}

Keywords: Deployment, Detection, Collaboration, Multi-Agent System, LMS, Wireless Sensor Networks

\section{INTRODUCTION}

Learning management system is a learning system application which enables administrators to track students' assessments and report their learning activities automatically (Ellis, 2009). This system can support multiple users accessing it. Designers can thus create digital learning contents which are stored in a database to be reused and managed to improve students' learning behaviors.
Almost all the new emerging technologies promise efficiency and enhancement of the overall system's performance. The collaboration of various components and integration techniques are very crucial concepts that help to design complex and effective systems (Sardis et al., 2011) for sharing scarce resources and reducing communication costs. Distributed sensor nodes are devices which are installed in an area or field for sensing the environment behavior Corresponding Author: Mwinyi, A.K., Department of Computer and Communication Systems Engineering, Faculty of Engineering, Universiti Putra Malaysia, 43400 UPM Serdang, Selangor Darul Ehsan, Malaysia 
(Patel and Jain, 2009). The growing numbers of Sensor Networks (SNs) applications in different disciplines are part of the motivation to introduce them in our research project. Other part of the motivation for using SNs is due to the proliferation of very small and reasonably priced sensors in the market today (Vinyals et al., 2011). As explained by Estrin et al. (1999), sensors can be used to monitor a wide spectrum of different conditions; among them is the presence or absence of certain objects in the environment. The presence of processing power, memory and wireless capabilities in sensor devices allow the sensors to integrate with MAS and hence increase LMS automation and adaptability.

Currently, the literature on LMS reveals that no project to the best of our knowledge has employed MAS that contains sensor nodes or devices to solve the students' location identification problem, once the students reach the LMS network coverage of LMS. Therefore, our research project deals with detecting and identifying the locations of the students in LMS domain environment. By monitoring the students' attendance, the students' performance in the classes can be enhanced. As the main goal of any LMS system is to provide online services to facilitate effectively classroom teaching (Meenakumari and Antony, 2013), we feel that there is a need to LMS to monitor our students' attendance in the classrooms in order to increase impact of LMS. This system in turns can improve the quality of education in institutions of higher learning.

The purpose of integrating Wireless Sensor Networks (WSN) with MAS is to widen the utilization of LMS as an online system with new features in order to make LMS applicable in online and offline classes by detecting the locations of students in LMS domain. This feature in LMS can be added by utilizing agents that contain sensors to sense the students' learning devices (e.g., smartphones, notebook computers, laptops and desktop computers) in LMS domain of a university's network coverage area. In addition, this operation of sensing the students' devices can be done by the collaboration of sensors and agents. Furthermore, the sensors can perform the overall monitoring of the students in LMS.

Base on the literature, this future LMS system with student detection capability is a promising technique which can be implemented in any of the two main categories of LMS (i.e., proprietary and open source LMS). The combination of sensors and MAS can be used to detect the intruders for security reasons (Marsh et al., 2004). Therefore, this combination can also be used to detect the students' locations in the learning process.

\section{MULTI-AGENT SYSTEM WITH WIRELESS SENSOR NETWORKS}

In recent years, WSNs have found their places in real-world appliances and have been extensively used to facilitate many practical applications including remote monitors, discovery and detection of significant physical phenomena (Marsh et al., 2004). The advancement of WSN technologies has gained momentum due to the huge progress in the development of MicroElectromechanical Systems (MEMS) technology and miniaturization of digital and electronic devices such as microprocessors (Marsh et al., 2004). These low cost and tiny devices are deployed on a large scale and in a distributed fashion to gain knowledge of the target areas under scrutiny. These devices have to perform complex computation tasks on physical quantities learned, before transmitting the information to intended destinations or end base stations. The physical quantities include temperature, humidity, light intensity, pressure and noise level (Singh et al., 2010). Advantages of using WSN for human development cannot be denied; however, this technology still faces setbacks with challenges that require innovative techniques and models in terms of its software and hardware.

One of the way to solve some software problems inherent in WSN's nodes and sensor nodes is to integrate WSN with MAS (Wood and Stankovic, 2002; Marsh et al., 2004). MAS provides room for sensor nodes to operate more intelligently by scrutinizing their physical environments and relaying information to the base stations or otherwise sharing with its neighbors for more decision making processes. According to Baig (2012), Multi-Agent (MA) constitutes numerous agents working together to accomplish certain task in which each agent is in charge of a specific tasks in the network. These tasks can be data discovery, analysis and filtering. Due to the design of the system, one or many agents can work together regularly to accomplish certain tasks and achieve the desired objectives of the system. MA is an intelligent piece of software or dedicated hardware invented for intended functionality which is capable of executing an event autonomously without the need of another entity's support (Guijarro et al., 2008).

Studies have been published regarding potential implementation of MAS in the sensor network's environment. As agents are capable of working independently to achieve the target objectives, these studies have used such capabilities to enhance the functionality of the WSN. Therefore, WSN with MA can 
not only monitor and detect the moving or stationary objects around the local vicinity, but also have a powerful paradigm to establish a significant controlling situation whereby the decisions may be made while ensuring that the global characteristics of the network are maintained to achieve WSN goals. As explained by Rogers et al. (2009), the mapping of MA technology into WSN is a complicated task as the latter does not describe how to solve the natural starving issues surrounding WSN, in terms of resources computation and processing, storage and communication in a highly dynamic environment. However, research advancements and recent contributions manage to make use of the agents' system in the field of WSN (Farinelli et al., 2008; Corkill et al., 2007).

Recent works on the integration of MAS in sensor network is based on power management to enhance the decision making process by incorporating intelligent behavior of an agent into WSN. Corkill et al. (2007) conducted a research using the technique of manipulating the sensor node's radio to involve the agents to make a collective decision to reduce energy consumption. As noted by Hill (2003), the sensor's radio is one of the sources of extracting node energy. Therefore, efforts have to be made to reduce transmission and reception activities.

Experiments with intruder detectors were conducted by Marsh et al. (2004) to compare two transmission schemes with intelligent agents software. The results revealed that power saving agents based on the schemes achieved almost 91\% (platform 2) of accuracy in data transmission compared to $71 \%$ (platform 3) of accuracy in saving energy by reducing transmission using sampling rate. In minimizing the number of transmissions by using well chosen sampling rate, the overall power consumption is saved and the accuracy of detection in the system is improved by boosting the frequency of detection. Table 1 illustrates the results as described earlier.

Table 1. Performance analysis by using different agent model

\begin{tabular}{|c|c|c|}
\hline $\begin{array}{l}\text { Measurement } \\
\text { unit }(\mathrm{Hz})\end{array}$ & Value & Agent model \\
\hline $\begin{array}{l}\text { Transmission } \\
\text { frequency }\end{array}$ & $\begin{array}{l}\text { Number of } \\
\text { transmissions }=153 \\
\text { and events detected } \\
=17 \text { per sec } \\
\text { Number of transmissions } \\
=64 \text { and events detected } \\
=20 \text { per sec }\end{array}$ & $\begin{array}{l}\text { Platform } 3 \\
\text { (sample every } 2 \mathrm{~s} \text { ) } \\
\text { Platform } 2 \\
\text { (sample every } 8 \mathrm{~s} \text {, } \\
\text { if ROC }<50 \text {,or } 0 \text {. } \\
5 \mathrm{~s} \text { if ROC }>50 \text { ) }\end{array}$ \\
\hline
\end{tabular}

ROC: Rate of Change of light level over time

\section{SENSOR NETWORKS ROUTING PROTOCOLS}

The Sensor Networks (SNs) is built up with inexpensive sensor nodes with different computational capabilities and powered by either battery or electricity (Marsh et al., 2004; Akyildiz et al., 2002). The improvement of SNs is due to advanced technologies used in building sensor nodes. These nodes have been widely used and are positioned in various areas in the sensing of physical phenomena (like temperature and light) and according to size. Furthermore, they can be easily set up in various environments, e.g., inside a room or outside.

To route data to the destination in the sensor networks, hundreds of sensor nodes are needed to work in a collaborative way under particular Routing Protocols (RPs) according to the working environment. RPs used in conventional networks (fixed networks) cannot be directly applicable to WSNs which normally work under unreliable wireless connection and hence has only partially or no infrastructure for routing the data. Therefore, numerous RPs have been created and implemented in WSNs for fixed and dynamic networks. In addition, various studies oin WSNs have classified WSNs and RPs in different classifications and taxonomies (Singh et al., 2010; Goyal and Tripathy, 2012; Biradar et al., 2009) due to their network's structure and needs.

The first Routing Protocol (RP), among the classifications is called Flat-Based Routing Protocol (FBRP) that comprises sensor nodes with similar responsibilities and roles (Karkazis et al., 2012). The second type of RP is called Hierarchical-Based Routing Protocol (HBRP) that consists of SNs in which each node has a responsibility to accomplish in the cluster arena (i.e., whether outside or inside of the cluster) such as LEACH (Heinzelman et al., 2000). The third RP is called Location-Based Routing Protocol (LBRP) that consists of sensor nodes with complete information of the locations or methods to identify their positions and hence are capable of routing the data to the destinations (Wood and Stankovic, 2002; Son et al., 2003).

Due to the working environment of the system, we have selected LBRP to incorporate into the learning system which encompasses MAS with sensors to identify the locations of the students in LMS because of the advantages highlighted earlier. Furthermore, the choice of merging the sensor networks in our research project is also due to the advantages of sensor technology paradigm (i.e., sensing technology) over other technologies as mentioned by Vinyals et al. (2011). Table 2 depicts the advantages of SNs as compared to other monitoring or sensing technologies. 
Table 2. Differences between sensor network and other monitoring technologies

\begin{tabular}{|c|c|c|}
\hline Feature & Sensor network & $\begin{array}{l}\text { Alternative } \\
\text { technology }\end{array}$ \\
\hline Sensor & $\begin{array}{l}\text { Low cost, } \\
\text { low power }\end{array}$ & $\begin{array}{l}\text { Expensive, } \\
\text { power consuming }\end{array}$ \\
\hline Coverage Area & Wide area & Small-size areas \\
\hline Monitoring & $\begin{array}{l}\text { Remote and/or } \\
\text { hostile environment }\end{array}$ & Highly controlled \\
\hline Robustness & $\begin{array}{l}\text { Fault-tolerant and } \\
\text { robust to node failure }\end{array}$ & Non-robust \\
\hline Invasiveness & Non-invasive & Invasive \\
\hline Data acquisition & $\begin{array}{l}\text { Irregularly } \\
\text { sampled datasets }\end{array}$ & $\begin{array}{l}\text { Regularly } \\
\text { sampled datasets }\end{array}$ \\
\hline Architecture & Distributed & Centralistic \\
\hline
\end{tabular}

\section{COLLABORATION OF MULTI- AGENT SYSTEM}

Multi-Agent System (MAS) basically contains heterogeneous agents to achieve certain goals or responsibilities (De Oliveira et al., 2006). In addition, MAS as proposed in various applications is a promising technology paradigm that comprises attractive characteristics like autonomous, intelligent and proactive which can be applied in software engineering and other disciplines for developing various systems of different complexity or applications (Zambonelli et al., 2003).

In order to show the importance of MAS, Talib et al. (2012) presented the security issue in cloud computing by incorporating MAS for securing Cloud Data (CD). They introduced a new security access control formula called Formula-Based Cloud Data Access Control (FBCDA). MAS's architecture that was presented consisted of two types of agents: Cloud Service Provider Agent (CSPA) which could provide access to the cloud resources and Cloud Data Confidentiality Agent (CDConA) which was responsible for formulating new access control for Cloud Data Storage (CDS).

Furthermore, Ogunnusi and Razak (2013), have introduced a fault-tolerant distribution security protocol for distributed mobile agents which is part of MAS entities to reduce the network intrusion attacks. In this, Ogunnusi and Razak (2013) utilized wireless local area network (WLAN) environment to detect the intrusive packets in the domain. This new distribution protocol for attach detection has includes various agents: Mobile agent, agent server and backup agent server. All these agents are working collaboratively with other components like, certification authority, security domain, messaging system, execution platform and keystore in order to ensure security of collaborating mobile agents from any possible attacks while migrating to the execution platform.

For MAS to work efficiently in any platform, there must be a communication and sharing of knowledge to accomplish the goals and tasks. When the agents collaborate and share knowledge, they do so within a group of agents with different capabilities to solve a set of problems. Nor et al. (2009) mentioned three types of knowledge: Organizational knowledge, managerial knowledge and technical knowledge that can be incorporated into the groups in the society or community to share information. MAS has been shown to contain different agents with different capabilities to achieve flexibility and enhance the interaction of LMS. MAS comprises a diversity of agents with different capabilities that can be used to predict the locations of users or a students as in our case, based on their device profiles and locations of the sensors which are stored in the databases.

MAS can offer various means of collaboration (Wooldridge and Jennings, 1995), among different types of agents in the system. Agents are capable of collaborating with other agents not only for exchanging data, but also for helping one another to perform certain tasks. This can be seen also from the model developed by Perez and Uresti (2014), where a number of agents are working together to predict the opponent next move. The model has been illustrated by an experiment with the RoboCup 2D Soccer Simmulator. In our research project the agents used had to collaboratively make decisions based on the locations of the students in LMS system. In addition, for knowledge sharing and interaction, MAS has to work together to find solutions or solve social or business problems, as individual agents have incomplete capabilities to solve complex problems (Sajja, 2008). Therefore, there is a need for MAS to work together and to share information that maximum performance of the system can be attained.

To show the importance of using MAS to improve the performance of the system, Boulaalam et al. (2013) have conducted a research that involve mobile agents to accelerate the new product development process. In their study that based on Auto ID, Boulaalam et al. (2013) have incorporated mobile multiagent system technologies, to improve the innovation process in the enterprise. In this proposed architecture of intelligent product, innovation can be improved by introducing the new product generation by utilizing MAS before the end of the ex-product version. 


\section{LMS WITH MAS SYSTEM ARCHITECTURE}

This section briefly explains the new system architecture model that comprises a number of sensor nodes and MAS within LMS coverage range. All sensor nodes make use of location detection technique which is GPS, in order for the sensor nodes to have a complete knowledge of their locations in LMS domain. Using wireless communication links, the sensor nodes create connected graph (ad hoc) among themselves and the Cluster Head Sensors (CHS) which is a sensor node with additional capabilities like power, memory and processing features which are differentiated from other sensors of WSNs. This node uses different types of protocols to exchange messages with other nodes.In this new architecture, Geographic Routing Protocol (GRP) is utilized to provide routing functionality among the nodes in WSNs and CHS. Furthermore, LBRP of geographic type is desired because of its capability of supporting scalability and mobility with the least routing overhead (Karkazis et al., 2012).

Among the agent types in this architecture are information agents in the sensor nodes which have the role of answering the queries from CHS about the data that have been manipulated and identified in their coverage area. Each sensor has its coverage area which is used to identify the users in that area. Currently, there are agents' technologies specifically to develop and create MultiAgents Platform (MAP) like JADE, JACK and JASON.

In various cases, WSNs are deployed for monitoring or sensing the discovery of environmental activities like detecting intruders in the system for security issues. In our case we utilize the combination of MAS with sensors to identify the students' locations in LMS. This situation can be realized by measuring the signal strength of a student's learning device, then the agents will collaboratively exchange messages using Agent Communication Language (ACL) and finally making decisions based on the exactly location of the student. In addition, the notified agent are also engaged in answering the queries on the status of the student, based on the information read by the agent in the student's learning device, upon authentication by LMS server. Hence, finally the notification agent can alert the student based on his or her location in LMS domain.

Figure 1 depicts the system architecture of LMS which deploys sensors that contain software agents for location detection.

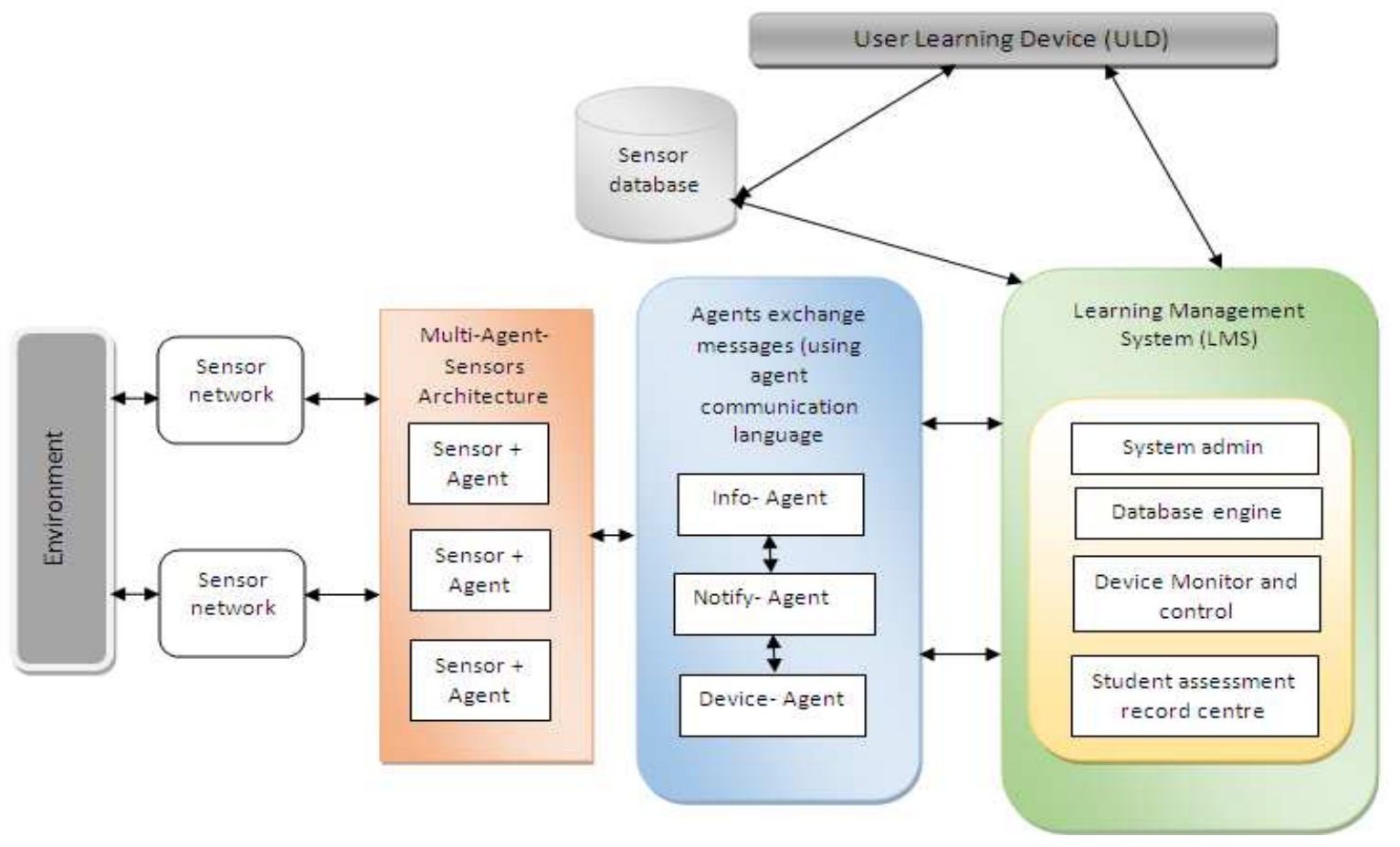

Fig. 1. System architecture involved multi-agents with sensors and system modules. Adopted from (Sardis et al., 2011) with redrawing based on modifications 
Table 3. Comparison with other LMS systems

\begin{tabular}{lllllll}
\hline Feature & Blackboard & Desire2Learn & eCollege & Moodle & Sakai & ATutor \\
Type of business & proprietary & proprietary & proprietary & open source & $\begin{array}{l}\text { open source } \\
\text { open source }\end{array}$ \\
\hline Student detection & $X$ & $X$ & $X$ & $X$ & $X$ & X \\
Interoperability & $\sqrt{ }$ & $\sqrt{ }$ & $\sqrt{ }$ & $\sqrt{ }$ & $\sqrt{ }$ & $\sqrt{ }$ \\
Accessibility & $\sqrt{ }$ & $\sqrt{ }$ & $\sqrt{ }$ & $\sqrt{ }$ & $\sqrt{ }$ & $\sqrt{ }$ \\
Usability & $\sqrt{ }$ & $\sqrt{ }$ & $\sqrt{ }$ & $\sqrt{ }$ & $\sqrt{ }$ & $\sqrt{ }$ \\
Interaction & $\sqrt{ }$ & $\sqrt{ }$ & $\sqrt{ }$ & $\sqrt{ }$ & $\sqrt{ }$ \\
Group work & $\sqrt{ }$ & $\sqrt{ }$ & $\sqrt{ }$ & $\sqrt{ }$ & $\sqrt{ }$ \\
Discussion forum & $\sqrt{ }$ & $\sqrt{ }$ & $\sqrt{ }$ & $\sqrt{ }$ & $\sqrt{ }$ \\
Administrative issue & $\sqrt{ }$ & $\sqrt{ }$ & $\sqrt{ }$ & $\sqrt{ }$ & $\sqrt{ }$ \\
SCORM compatibility & $\sqrt{ }$ & $\sqrt{ }$ & $\sqrt{ }$ & $\sqrt{ }$ & $\sqrt{ }$ \\
Interactive feature & $\sqrt{ }$ & $\sqrt{ }$ & $\sqrt{ }$ & $\sqrt{ }$ & $\sqrt{ }$ \\
Course development & $\sqrt{ }$ & $\sqrt{ }$ & $\sqrt{ }$ & $\mathrm{X}$ & $\sqrt{ }$ \\
Synchronization & $\sqrt{ }$ & $\sqrt{ }$ & $\sqrt{ }$ & & & $\sqrt{ }$ \\
\hline
\end{tabular}

In this scenario, sensors nodes are responsible for sensing the incoming students' devices to the campus environment and then report these findings to MAS which in turn communicate with one another in order to reach an agreement on the exact location of the devices in LMS network coverage area. Finally, LMS system interacts with the sensor database to identify the location of the students in the HLI domain.

\section{COMPARISON WITH OTHER LMS SYSTEMS FEATURES}

In this section, the comparison of selected commercial and open sources in LMS is presented. The results are briefly summarized in Table 3 which includes six commonly used LMS providers in the world (Berking and Gallagher, 2013; Aberdour, 2007). Among the selected LMS are three proprietary and three open sources. In this research project, we involved the main functionalities or features which can be found in each of the aforementioned LMS.

In this investigation as shown in Table 3 involving the world's leading commercials and open sources LMS, no previous studies to the best of our knowledge have incorporated student detection features to support functionalities or features package. From our point of view, this proposed LMS system can help to develop well blended LMS system to track students' attendances in Face-to-Face (F2F) classes in HLI while using online service.

\section{CONCLUSION}

The detection of students' locations in LMS is crucial in maximizing the students' overall performance, improving their attendance and eventually increasing their activities in HLIs. Sensory data are collected and processed by CHS before transmitting to LMS database for comparing and making the final decision on a student's location. As sensor nodes are deployed and installed in distributed approach, the multi-agents themselves detect a student's location autonomously. This study has presented a system architecture that integrates MAS with sensor networks to easily monitor students in LMS. As mentioned above, this proposed learning system can be only applicable in the systems that support blended mode of teaching where students are suppose to reach certain percentage of class or lab attendece. Future works can involve real-world data to test the applicability of this new system architecture and also to improve security issue in LMS.

\section{ACKNOWLEDGEMENT}

The researchers of this study would like to acknowledge the Exploratory Research Grant Scheme (ERGS) from the Ministry of Education Malaysia 2012/2014 and the GIPP-UPM: Intuitive learning environment in higher institutions 2012/2014, for financial support.

\section{REFERENCES}

Aberdour, M., 2007. Open source learning management systems. Epic.

Akyildiz, I.F., W. Su, Y. Sankarasubramaniam and E. Cayirci, 2002. Wireless sensor networks: A survey. Comput. Netw., 38: 393-422. DOI: 10.1016/S13891286(01)00302-4

Baig, Z.A., 2012. Multi-agent systems for protecting critical infrastructures: A survey. J. Netw. Comput. Applic., 35: 1151-1161. DOI: 10.1016/j.jnca.2012.01.006 
Berking, P. and S. Gallagher, 2013. Choosing a learning management system. Serco Services, Inc.

Biradar, R.V., V.C. Patil, S.R. Sawant and R.R. Mudholkar, 2009. Classification and comparison of routing protocols in wireless sensor networks. Special Issue Ubiquitous Comput. Security Syst., UbiCC J., 4: 704-711.

Boulaalam, A., E.H. Nfaoui and O.E. Beqqal, 2013. Intelligent product based on mobile agent to accelerate the new product development process. J. Comput. Sci., 9: 856-865. DOI: 10.3844/jcssp.2013.856.865

Corkill, D.D., D. Holzhauer and W. Koziarz, 2007. Turn off your radios! environmental monitoring using power-constrained sensor agents. Proceedings of the 1st International Workshop on Agent Technology for Sensor Networks, (TSN' 07), Honolulu, Hawaii, pp: 31-38.

De Oliveira, R., S. Gomes, H. Silva, A. Bittencourt and E.B. Costa, 2006. A Multi-agent based framework for supporting learning in adaptive automated negotiation. ICEIS, 4: 153-158.

Ellis, R.K., 2009. A field guide to Learning Management Systems. Learn. Cir. Am. Soc. Train. Dev.

Estrin, D., R. Govindan, J. Heidemann and S. Kumar, 1999. Next century challenges: Scalable coordination in sensor networks. Proceedings of the 5th Annual ACM/IEEE International Conference Mobile Computing Networking, Aug. 15-19, Washington, USA., pp: 263-270. DOI: 10.1145/313451.313556

Farinelli, A., A. Rogers, A. Petcu and N.R. Jennings, 2008. Decentralised coordination of low-power embedded devices using the max-sum algorithm. Proceedings of the 7th International Joint Conference on Autonomous Agents and Multiagent Systems, May 12-16, Richland, pp: 639-646.

Goyal, D. and M.R. Tripathy, 2012. Routing protocols in wireless sensor networks: A survey. Proceedings of the 2nd International Conference Advanced Computing Communication Technologies, Jan. 7-8, IEEE Xplore Press, Rohtak, Haryana. DOI: 10.1109/ACCT.2012.98

Guijarro, M., R. Fuentes-Fernandez and G. Pajares, 2008. A multi-agent system architecture for sensor networks. State Art Sens. Technol. Spain, 9: 1024410269. DOI: $10.3390 / \mathrm{s} 91210244$
Heinzelman, W.R., A. Chandrakasan and H. Balakrishnan, 2000. Energy-efficient communication protocol for wireless microsensor networks. Proceedings of the 33rd Annual Hawaii International Conference System Sciences, Jan. 4-7, IEEE Xplore Press. DOI: 10.1109/HICSS.2000.926982

Hill, J.L., 2003. System Architecture for Wireless Sensor Networks. PhD Thesis, University of California.

Karkazis, P., H.C. Leligou, T. Orphanoudakis and T. Zahariadis, 2012. Geographical routing in wireless sensor networks. International Conference Telecommunications Multimedia, Jul. 30-Aug. 1, IEEE Xplore Press, Chania, pp: 19-24. DOI: 10.1109/TEMU.2012.6294717

Marsh, D., R. Tynan, D. O'Kane and G.M.P. O'Hare, 2004. Autonomic wireless sensor networks. Eng. Applic. Artif. Intell., 17: 741-748. DOI: 10.1016/j.engappai.2004.08.038

Meenakumari, J. and B. Antony, 2013. Assessing the essential features of ICT-based LMS for performance enhancement. Int. J. Res. Manage. Technol.

Nor, M.Z.M., R. Abdullah, M.H. Selamat and M.A.A. Murad, 2009. Knowledge sharing interactions in collaborative software maintenance environment. Proceedings of the International Conference Computer Technology and Development, Nov. 1315, IEEE Xplore Press, pp: 201-205. DOI: 10.1109/ICCTD.2009.66

Ogunnusi, O.S. and S.A.D. Razak, 2013. Research on security protocol for collaborating mobile agents in network intrusion detection systems. Am. J. Applied Sci., 10 : 1638-1647. DOI: 10.3844/ajassp.2013.1638.1647

Patel, R.B. and D. Jain, 2009. A multiagent system for distributed sensor networks. Proceedings of the International Conference Advances in Computing, Control and Telecommunication Technologies, Dec. 28-29, IEEE Xplore Press, Trivandrum, Kerala, pp: 823-826. DOI: 10.1109/ACT.2009.208

Perez, A.B.G. and J.A.R. Uresti, 2014. Strategy patterns prediction model. J. Comput. Sci., 10: 73-84. DOI: $10.3844 /$ jcssp.2014.73.84

Rogers, A., D.D. Corkill and N.R. Jennings, 2009. Agent technologies for sensor networks. IEEE Intell. Syst., 24: 13-17. 
Sajja, P.S., 2008. Multi-agent system for knowledgebased access to distributed databases. Interdisciplinary J. Inform. Knowl. Manage., 3: 1-9.

Sardis, E., N. Matsatsinis and A. Doulamis, 2011. Sensor networks and multi-agents in industrial workflows. Int. J. Mac. Learn. Comput., 1: 205-212.

Singh, S.K., M.P. Singh and D.K. Singh, 2010. Routing protocols in wireless sensor networks-A survey. Int. J. Comput. Sci. Eng. Survey, 1: 63-83.

Son, S., B.M. Blum, T. He and J.A. Stankovic, 2003. IGF: A state-free robust communication protocol for wireless sensor networks. Tec. Report Depart. Comput. Sci. Univ. Virginia.

Talib, A.M., R.B. Atan, R. Abdullah and M.A.A. Murad, 2012. Towards a comprehensive security framework of cloud data storage based on multi agent system architecture. J. Inform. Security, 3: 295-306. DOI: $0.4236 / \mathrm{jis} .2012 .34036$
Vinyals, M., J.A. Rodriguez-Aguilar and J. Cerquides, 2011. A survey on sensor networks from a multiagent perspective. Comput. J., 54: 455-470. DOI: $10.1093 /$ comjnl/bxq018

Wood, A. and J.A. Stankovic, 2002. Denial of service in sensor networks. Comput., 35: 54-62. DOI: 10.1109/MC.2002.1039518

Wooldridge, M. and N.R. Jennings, 1995. Intelligent agents: Theory and practice. Knowl. Eng. Rev., 10: 115-152. DOI: 10.1017/S0269888900008122

Zambonelli, F., N.R. Jennings and M. Wooldridge, 2003. Developing multiagent systems: The Gaia methodology. ACM Trans. Software Eng. Methodol., 12: 317-370. DOI: $10.1145 / 958961.958963$ 